\title{
Criminologie
}

\section{La nouvelle criminologie et la délinquance}

\section{Denis Szabo}

Volume 8, numéro 1-2, 1975

Délinquance juvénile au Québec

URI : https://id.erudit.org/iderudit/017045ar

DOI : https://doi.org/10.7202/017045ar

Aller au sommaire du numéro

Éditeur(s)

Les Presses de l'Université de Montréal

ISSN

0316-0041 (imprimé)

1492-1367 (numérique)

Découvrir la revue

Citer ce compte rendu

Szabo, D. (1975). Compte rendu de [La nouvelle criminologie et la délinquance] Criminologie, 8(1-2), 175-188. https://doi.org/10.7202/017045ar d'utilisation que vous pouvez consulter en ligne.

https://apropos.erudit.org/fr/usagers/politique-dutilisation/ 


\section{LA NOUVELLE CRIMINOLOGIE}

ET LA DELINQUANCE

Note de lecture 
Périodiquement, il y a des livres qui remettent en cause, d'une manière globale, les interprétations habituelles dans une discipline. D'ordinaire, ils suscitent quelques approbations, mais surtout beaucoup de résistance puisqu'ils n'épargnent pas de leurs critiques les prédécesseurs. C'est un phénomène fort sain en soi : on a tendance à s'enliser dans le conformisme d'explications partielles sans se poser ou reposer des questions fondamentales qui légitimement, en fin de compte, la démarche intellectuelle de tout homme de science.

La criminologie fut enfin servie, après les autres sciences de l'homme, et bien servie pour se faire dire ses quatre vérités! Les «criminologues critiques > ont finalement trouvé en Taylor, Walton et Young ${ }^{1}$, des porte-parole bien articulés, agressifs à souhait, exprimant bien les préoccupations de tous ceux qui refusaient la perspective positiviste et réformiste qui caractérise la vaste majorité des travaux criminologiques du passé et du présent.

Nous saisissons avec un vif plaisir l'occasion qui s'offre de commenter pour nos lecteurs ce livre et de présenter quelques réflexions critiques que les charges à fond des auteurs appellent de la part de quelqu'un qui appartient à une autre famille d'esprit. Les études de la délinquance juvénile sont touchées autant par ces réflexions que le reste de l'« opus » et de la \& praxis * criminologiques. Il n'y a donc rien d'inusité d'en parler dans le présent numéro car les critiques des auteurs s'adressent à tous ceux qui œuvrent dans le chantier de notre discipline.

1. Ian Taylor, Paul Walton et Jack Young, The New Criminology, Routledge et Kegan Paul, 1973. 
Pourquoi le ton ultra-critique des comptes rendus consacrés à ce livre ? On le comprend bien en lisant la préface d'Alvin Gouldner qui insiste pour que les couleurs soient abattues sans ambages. L'intention des auteurs est de briser la conspiration du silence entretenue par des générations de criminologues qualifiés de «traditionnels » : by launching a deliberate discourse concerning the general social theory usually only tacit in specialized work on crime and deviance » et «by liberating technical « topics \$ into a newly enlivening, larger, more reflexive critique $(\mathrm{p} . \mathrm{x})$. L'introduction - mais il serait plus exact de dire la réintroduction des études criminologiques dans le discours sociologique général devait apparaître tôt ou tard. Elle n'est, toutefois, pas une opération de nature purement théorique. Elle affecte directement la praxis criminologique en soulignant la nécessité d'accepter * the reality of deviance, that has a capacity to explore its Lebenswelt, without becoming the technician of the $\$$ Welfare $\gg$ State and its zoo-keepers of deviance (p. XIv).

L'intention du livre clairement définie ainsi : la mission critique libératrice annoncée, nos auteurs abordent en huit chapitres la tâche ardue de résumer et de critiquer le «corpus > de la tradition criminologique.

Les deux premiers chapitres constituent l'entrée en matière : 4Classical Criminology and the Positivist Revolution 》. Partant de Beccaria, les auteurs analysent la réaction positiviste que l'école classique de droit pénal a suscitée à la fin du XIXe siècle. Remontant jusqu'à Lombroso et Ferri, ils procèdent à une critique vigoureuse des auteurs contemporains qu'ils situent dans la tradition positiviste : Eysenck, Trasler, parmi les psychologues, accessoirement Tappan, Wilkins, Sellin et Wolfgang, parmi les sociologues. Au passage, la critique du positivisme par d'autres (comme Matza, par exemple) est vivement contestée. Les postulats de la position positiviste sont résumés avec netteté : «Thus from the initial three premises of the scientific method - measurement (quantification), objectivity (neutrality), and causality (determinism) - are derived a number of postulates : a consensus view of the world, a focus on the criminal act, a reification of the social world, a doctrine of non-responsibility for actions, the inapplicability of punishment, 
and the faith in the superior cognitive ability of the scientific expert (p. 23). Le lien entre la théorie scientifique et les attitudes sociales des chercheurs est bien mis en évidence.

Le chapitre III consacré à Durkheim, est présenté comme une rupture avec l'«analytical individualism . Effectivement, le sociologue français fut le seul, parmi les grands fondateurs de la sociologie, à s'être préoccupé d'une manière précise de l'explication du crime comme phénomène social faisant partie de l'univers social total. Ni Weber, ni Simmel, ni Pareto, ni Marx n'ont réservé dans leurs réflexions ou dans leurs investigations une place autre que périphérique à l'étude de la criminalité. La tradition française de "science morale », l'étude privilégiée des mœurs a probablement influencé Durkheim en l'incitant à examiner les fondements sociologiques de la "déviation» des normes sociales et légales. Cette première théorie générale de la sociologie du crime et de la déviance, qui fut à l'origine de l'école structurelle-fonctionnelle dominant la sociologie presque jusqu'à nos jours (un bel exemple qu'ils ne rappellent pas est Crime and Custom in Savage Society de Malinowski, 1926), est jugée insuffisante par les auteurs car bien que représentant une rupture salutaire avec l'individualisme analytique des positivistes, cela est obtenu « at the expense of erecting an incomplete picture of society, and, in particular, at the expense of ambiguity over the questions of rationality, purposiveness and socialization in divided societies ( $\mathrm{p}$. 90). Il est bien exact que Durkheim fut fondamentalement un rationaliste et quelque peu organiciste avec son insistance sur la suprématie de la conscience collective sur la conscience individuelle.

La poussée (breakthrough) théorique de Durkheim a enfin permis le développement d'une série d'études sociologiques consacrée à la criminalité, principalement aux États-Unis. Merton a fait la contribution théorique la plus importante en reformulant, d'une manière originale, la théorie de l'anomie développée par Durkheim à propos de l'étiologie du suicide. Cloward et Ohlin furent parmi les disciples les plus originaux et les plus féconds du sociologue de Columbia : le nombre des études qu'ils ont inspiré dans les années 60 est impressionnant. On n'insiste jamais suffisamment sur la véritable renaissance de la sociologie criminelle que ces études représentent dans une criminologie * traditionnelle ", massivement dominée par les idées et les méthodes des Gluecks. L'école de Chicago, branche du positivisme naturaliste 
représenté par les travaux de Park, Burgess et Ogburn, a également suscité d'amples moissons. Finalement, la tendance interactionniste - Sutherland et ses disciples figurent, par ailleurs, en bonne place parmi les épigones de Durkheim - s'appuyant davantage sur la psychologie sociale de Mead, a produit un intérêt croissant à partir du milieu des années 60 dans le sillage d'Albert Cohen : Becker, Kitsuse, Lemert sont examinés et critiqués.

Pour nous auteurs, toutes ces recherches manquent, en dernière analyse, d'une théorie cohérente de la «déviance ». Elles ne tiennent guère compte de la structure du pouvoir et des intérêts. Ils notent qu'un « relevant theory of deviancy must treat the causal variables - motivation and reaction - as determinate and as part of a total structure of social relationships. [...] We see that the institution of private property, in a stratified and inequitable society, divides men from men as owners and non-owners. It is in the light of this division that the activities of thieves, police, magistrates and property-owners become explicable $\gg$ (p. 170). Toute une série de conduites «déviantes », celles de voleurs, d'espions industriels, d'étudiants rebelles, ne peuvent recevoir une explication satisfaisante dans la perspective interactionniste. Il manque, en effet, « a detailed social history of the constraints, aspirations and meanings which inform and activate the actors $\gg$ (p. 170). De même que la théorie des opportunités différentielles explique mal le phénomène de militantisme noir, l'interactionnisme demeure insuffisant (falls short) de l'explication des conduites qui sont occasionnées par des données structurelles globales. Nos auteurs se proposent maintenant d'aborder « a structural sociology on the one hand (a sociology competent to deal with power and interest) and a sociology of motivation on the other (a sociology that can account for the way in which individuals give meaning to their acts), (p. 171).

Dans le chapitre consacré à l' American naturalism and phenomenology», la contribution de l'ethnométhodologie est examinée à partir de l'œuvre de Matza. La préoccupation épistémologique a dominé tout naturellement ce chapitre: quelle est la nature exacte du phénomène "déviance 》? Les écrits de Garfinkel, Cicourel, Sacks, etc., sont "critiquement » évalués et nos auteurs concluent que cette sociologie de la vie quotidienne are to be located within higher order life-plans. It is precisely these normative life-plans, world views, or ideologies which constitute the cement 
which provides the beliefs necessary for the maintenance of social system. [...] Life is not a game and only certain beliefs will sustain specific social systems 》 (p. 207-208). Finalement, * the differential availability of accounts to members is something which ethnomethodology cannot and does not study, yet it is precisely this problem which is at the basis of the distribution of motives which inform deviant behavior [...] in bracketing away the question of social reality, it does not allow of any description of the social totality we assert to be productive of deviance (p. 208). Si les ethnométhodologues ont renouvelé l'étude des normes et des valeurs, la plupart ne se sont pas engagés pour accepter une « sociologie normative ». Refusant la téléologie ailleurs qu'au niveau des interactions inter-individuelles, les ethnométhodologues demeurent bien en deçà des exigences de nos «nouveaux criminologues 》.

Le chapitre vir est consacré à Marx, à Engles et à leur disciple Bonger. La tâche des auteurs est difficile, car comme ils notent : -... one of the most telling features of Marx's statements on crime is their a-typicality when compared to the vast body of orthodox marxism» (p. 219). C'est la théorie générale de Marx qui permet de déduire des conclusions pertinentes pour la criminologie plutôt que des remarques spécifiques concernant la criminalité. Voici l'interprétation qu'ils donnent de cette théorie générale : " a full blown marxist theory of deviance... would be concerned to develop an explanation of the ways in which particular historical periods, characterized by particular sets of social relationships and means of production, give rise to attempts by the economically powerful to order society in a particular way... Who makes the rules and why? (p. 220). Seule une telle théorie permettrait enfin de rendre compte " that much deviance is in itself a political act, and that, in this sense, deviance is a property of the act rather than a spurious label applied to the amoral or the careless by agencies of political and social control \$ (p. 221).

Au terme de ce chapitre, les auteurs offrent une démarche marxiste dans la stratégie de la recherche criminologique : $I t$ would start with crime as a human action, as a reaction to positions held in an antagonistic social structure, but also as action taken to resolve those antagonisms. It would... involve a model... of the dialectics of human action, however, or for whatever reason they tend to be defined as 'criminal' in particular historical periods by the powerful... It would proceed to understand the relationship 
of criminal action, and an understanding of its dynamics, to human liberation» (p. 236). Voici donc l'unité intellectuelle de la théorie de l'action, aspiration fondamentale de toute pensée socialiste. Le crime résulte d'un conflit entre forces antagonistes, et seul par son replacement dans les conflits fondamentaux qui dominent la structure socio-économique des sociétés capitalistes qu'une analyse "correcte 》 de la criminalité peut avoir lieu. Le crime est l'expression de l'aspiration des oppressés pour contester ou secouer le joug de leur oppresseur.

Le dernier chapitre traite de new conflict theorists : Vold, Dahrendorf, Turk, Quinney, sont placés dans une perspective post ou néo-marxiste. C'est avec sympathie que les auteurs examinent la contribution de cette "école s. Néanmoins, celle-ci demeure insuffisante en regard des exigences de la «nouvelle criminologie ». Il leur manque le plus fondamental des prérequis (prerequisites) d'une théorie générale: la juste appréciation de l'action humaine. Pour chacun de ces auteurs, le fait criminel, l'homme criminel, demeure pathologique. Et nos auteurs soulignent que «such a conception undermines or understresses an alternative view of men as purposive creators and innovators of action 》 (p. 267). "The conflict approach is in danger of withdrawing integrity and purpose - or idiosyncracy - from men : and thus, is close to erecting a view of crime as a non-purposive (or pathological) reaction to external circumstances $\gg$ (p. 267). Tout sera donc encore trop mécaniciste, trop déterministe au gré de nos critiques : Comment sauver "l'authenticité \$ des actes et des intentions si les intérêts dominent si massivement les motivations ?

Quelle est maintenant la théorie des auteurs? Nous en avons eu un avant-goût en citant les passages significatifs de leur critique de la théorie des autres. Voyons la leur. Pour eux, « an adequate social theory would need to be free of the biological and psychological assumptions that have been involved in the various attempts to explain the actions of the men who do get defined and sanctioned by the state as deviant, and react against these definitions, in different historical circumstances $\gg$ (p. 268).

De plus, cette théorie doit rendre compte "the forms assumed by social control and deviant action in edeveloped , societies characterized... by the domination of a capitalist mode of production, by a division of labour involving the growth of armies of experts, social workers, psychiatrists and others who have been 
assigned a crucial role in the task of social definition and social control, and currently by the necessity to segregate out - in mental hospitals, prisons and in juvenile institutions - an increasing variety of its members as being in need of controls (p. 269). Dans une société capitaliste, qu'il soit ou non tempéré par la version socialiste de Welfare State, tout contrôle social fait partie de l'appareil de répression dont la mission est de maintenir l'ordre public au service des intérêts de la classe dirigeante. De ce fait, toute «science > qui est utilisée à cette fin a, objectivement, une fonction antisociale et répressive.

Le dernier alinéa du livre constitue la proclamation de foi finale : * For us... deviance is normal - in the sense that men are now consciously involved (in the prisons that are contemporary society and in the real prisons) in asserting their human diversity... The task is to create a society in which the facts of human diversity, whether personal, organic or social, are not subject to the power to criminalize (p. 282).

Nous espérons avoir donné un aperçu succinct mais fidèle du contenu du livre et indiqué ses orientations. L'exposé est toutefois forcément schématique et ne donne probablement qu'une idée fort superficielle de la richesse des aperçus théoriques, de la vivacité des arguments polémiques, de la générosité évidente de l'inspiration morale qui anime les auteurs. Je dirais sans ambages que j'ai éprouvé un vif plaisir - mêlé à une certaine irritation et à beaucoup d'inquiétude - à lire le livre (deux fois, pour les fins de ce compte rendu). Taylor, Walton et Young s'inscrivent dans la tradition iconoclastique qu'illustrent des auteurs comme Szasz, Michael et Adler, Andrewski (dont le Social Science as Sorcery, récemment publié à Londres, me procurait des sensations bien semblables à celles que j'ai éprouvées pour les meilleures pages du présent livre - bien que l'inspiration de cet auteur soit à l'opposé des protagonistes de la nouvelle criminologie -). Ils adoptent d'emblée une position ultra-critique à l'égard non seulement de la criminologie scientifique traditionnelle, mais également et peut-être plus encore à l'égard des autres tendances novatrices, voir marxistes, de la criminologie. 
J'adresse quatre reproches principaux aux auteurs : schématisme de l'exposé, assimilation de la théorie à l'idéologie, holisme peu nuancé et angélisme prophétique. Par la suite, je reconnais d'avance ma propre culpabilité dans la schématisme excessif de ma propre critique.

\section{Schématisme de l'exposé}

Il n'y a donc rien d'étonnant à ce que les critiques que nous avons citées aient rendu aux auteurs les pièces de leur monnaie : leur réaction fut largement négative. Mais cette réaction ne tient pas seulement de l'attitude hypercritique de nos auteurs. Se livrant à une réévaluation globalisant de l'apport criminologique au discours théorique contemporain, en cours dans les sciences humaines, les auteurs s'assignent une tâche bien périlleuse. Il est vrai que les écrits criminologiques ont été très pauvres en critique épistémologique. Néanmoins, ils rendent à tous un service insigne, en faisant la "sociologie de la criminologie s. Mais ne s'improvise pas qui veut en épistémologie et une critique à portée aussi ambitieuse que celle-ci demeure cruellement schématique et arbitraire aux yeux du spécialiste. Relevant les inconsistances et les simplifications abusives des auteurs dans le traitement de sujets aussi complexes et concernant en particulier leur critique sommaire de la neutralité scientifique, la remarque de Beyleveld (op. cit., p. 394-395) me paraît totalement justifiée. Comme tous les polémistes, ils dychotomisent arbitrairement ,appauvrissent ainsi lamentablement les sujets traités et donnent une fausse impression de rigueur à ce qui n'est qu'une manifestation d'un regrettable manichéisme intellectuel et moral. Cette même critique peut s'adresser à tous les chapitres : simplification et distorsion des arguments des auteurs discutés, lacunes considérables dans la documentation, qui se manifestent dans la sélection arbitraire d'ouvrages critiqués, une subordination permanente de la finalité de l'investigation scientifique à la finalité de l'action normative et politique.

Comme criminologue de langue française, je n'insisterai pas sur ce phénomène regrettable chez tant d'auteurs anglo-américains d'ignorer tout sur la littérature qui s'écrit dans d'autres langues. Les nouveaux criminologues partagent ces préjugés avec la plupart des autres auteurs. On peut bien ajouter aux « crimes, dénoncés par les radicaux (« sexism », " imperialism », etc.), l'ethnocentrisme indigne de vrais intellectuels. Mais lorsqu'on se pique 
d'être marxiste, donc historien autant que philosophe, économiste autant que sociologue, psychologue autant que critique social et homme d'action, il faut bien accepter d'être jugé à la hauteur de cette ambition si nettement proclamée.

Or, la compréhension des auteurs de l'histoire laisse perplexe. Les idées de Beccaria, notées au départ de ce livre et fils conducteurs de la pensée juridique jusqu'à nos jours, sont traitées " a-historiquement ». La référence à Angela Davis est immédiate, le contexte politique contemporain envahissant. Saleilles (traduit pourtant en anglais!) analysait avec finesse la confrontation de l'école classique de droit pénal avec le positivisme naissant. Le bénéfice de la compréhension de la perspective historique dans laquelle émergent Rousseau, Montesquieu et Beccaria n'est guère apparent à nos auteurs. Qualifier Tarde de positivisme (p. 17) est comme proclamer Sir Karl Popper ou Lord Bertrand Russel d'idéalistes... Et on pourrait glaner beaucoup de ces contresens dus à un trop rapide, trop polémique, trop simpliste traitement de données qui méritent et ont mérité, sous d'autres plumes, de bien plus justes et nuancées appréciations.

Rappelons, pour mémoire, que la foi dans la raison et dans la science s'opposait à l'arbitraire de la force brutale des régimes absolutistes. Justifié par une théologie ultramontaine, évidemment téléologique, l'ancien régime ne pouvait être contesté que par la proclamation du libre examen basé sur l'autorité de la recherche scientifique. Escamotant la profondeur des problèmes qui surgissent de l'analyse historique, le simplisme des auteurs contribue peu à un débat intellectuel sérieux.

\section{Assimilation de la théorie à l'idéologie}

La lacune que j'ai ressentie le plus péniblement concerne l'absence de toute référence à la praxis criminologique ou même socio-politique concrète. Le schématisme pamphlétaire des auteurs demeure toujours à un tel niveau d'abstraction et de généralité qu'aucune des propositions avancées n'apparaît testable ou susceptible d'être confrontée avec une praxis quelconque. Le plan final propose, que nous rappelions, qui pourrait être contre cela? Pourtant, dès qu'on entre dans les détails, on découvre le désaccord. Des références vagues aux liens entre réformisme, libéralisme et capitalisme ne peuvent pas être substituées à une analyse rigoureuse décrivant l'interaction entre les diverses idéologies scien- 
tifiques, la réalité socio-politique, socio-économique et socio-administrative. Cette absence de l'examen de la politique criminelle comme partie d'une politique sociale affaiblit beaucoup l'intérêt et la portée des arguments. L'analyse des relations entre les objectifs de la politique sociale de New Deal, du plan Beveridge, des régimes socio-démocratiques scandinaves, par exemple, reste entièrement à faire. Sans une telle étude, les affirmations des * nouveaux criminologues $>$ demeurent gratuites et ne sont pas, intellectuellement, recevables.

La confrontation entre l'idéologie (car il s'agit bien plus de cela que de théorie) et la praxis fait le plus cruellement défaut chez ces «néo-marxistes \ lorsqu'on considère la réalité sociopolitique, judiciaire et criminologique des pays professant l'idéologie marxiste-léniniste. De nombreux ouvrages possédant un haut degré de crédibilité scientifique, intellectuel et moral, existent. Ils permettent de voir comment la plupart des thèses avancées par les auteurs ont subi un intéressant test de réalité depuis 1917. Mais il manque, dans l'index entre Lemert et Levin, le nom de Lénine dont l'apport à la théorie et surtout à la praxis judiciaire marxiste fut tout de même notable. Ce n'est quand même pas Jackson, Cleaver, etc., émergeant des luttes des minorités américaines par l'égalité et témoignant par des écrits poignants de l'injustice ressentie, qui pourront faire mettre entre parenthèses l'univers décrit par Soljenytzine.

Cette réduction de la perspective historique à la perception qu'a d'un monde très partiel la génération baignée dans l'atmosphère de la société post-industrielle, de la guerre du Vietnam, et de la décolonisation du Tiers Monde, imprime peut-être la plus sévère limitation à ce livre. Suivant un mécanisme psycho-social bien connu, il sera absorbé et applaudi par ceux dont l'expérience et la sensibilité socio-politique est similaire à celles des auteurs. Comme tant d'autres ouvrages (le meilleur exemple étant celui de Reich, The Greening of America), il exprime une manifestationéclair (flash) du Zeitgeist, sans atteindre et sans contribuer au dialogue fondamental que l'humanité poursuit depuis l'aube des temps.

Pour ceux qui s'interrogent sur la dialectique fondamentale entre liberté et contrainte, ordre et changement, bien et mal, vice et vertu, ce livre reste de peu de secours. Nous avons libéralement 
cité des auteurs pour présenter avec justice leur position. La citation de ces quelques lignes de Burke peut être utilement mise en regard de leur philosophie de la liberté : « Men are qualified for civil liberty in exact proportion of to their disposition to put moral chain upon their own appetites... society cannot exist unless a controlling power upon will and appetite be place somewhere, and less of there is within, the more there is without. It is ordained in the eternal constitution of things that men of intemperate mind cannot be free... *

Le vocabulaire a sans doute vieilli; on a certes abusé des 4 chaînes > que volontairement ou involontairement les hommes ont imposées ou se sont imposées à l'exercice de leur liberté. II n'en reste néanmoins que le principe énoncé mérite la plus grande des attentions de la part de ceux qui s'occupent de la morale comme de la \& science , de l'homme.

\section{Holisme sans nuances}

Ceux qui ont choisi la * science , pour étudier et comprendre l'homme déviant ou criminel ne seront que peu avancés par les propositions de la * nouvelle criminologie \$. Pour ceux-là, rien ne peut remplacer le processus rigoureux qui consiste à tester les idées, les pratiques d'une manière standardisée et systématique, quelle que soit, par ailleurs, la méthode ou l'épistémologie choisie.

La division du travail entre * politiques > et \& scientifiques s, entre "moralistes > et «étaphysiciens ?, entre \& bureaucrates ? et \& intellectuels > a été obtenue au prix de trop longs efforts, de trop grands sacrifices et au bénéfice de trop évidents avantages, pour qu'on y renonce d'un cour léger : Cela ne veut pas dire que j'estime que cette division du travail ne va pas sans danger. Là aussi, un long catalogue de mise en garde, de cavéat s'impose. Mais, la subordination directe et immédiate de toute théorie, de toute pratique, de tout moyen, à des impératifs idéologiques n'ont produit que des régimes socio-politiques où l'intolérance règne en maîtresse et où il ne fait pas bon de vivre pour les hommes libres et responsables. L'histoire contemporaine présente de trop éloquents exemples qui illustrent ces propos. Les souvenirs sont dans toutes les mémoires et devront être dans celles de la génération qui a vécu Watergate. 


\section{Angélisme prophétique}

Ceux enfin qui sont des «déviants 》, des c criminels 》 ou qui s'en occupent soit par vocation, soit par nécessité, risquent de voir leur frustration encore exacerbée à la suite de la lecture de ce livre. La conscience morale de l'homme, en effet, ne se réduit pas à un simple reflet des luttes politiques du jour. Il sera bien trop simple de comprendre et d'agir sur la conduite déviante en fonction d'engagements idéologiques et d'actions politiques directes. Il me semble, au contraire, que les forces du mal sont vraiment à l'œuvre et dans l'histoire, et dans l'esprit et le cœur des hommes. Accepter cette donnée fondamentale de la condition humaine, la possibilité de la défaite comme celle de la victoire implicite dans le principe même de notre liberté d'agir, c'est faire preuve d'humanité et non seulement d'humilité.

La manière concrète dont l'homme et la société réagissent au triomphe du mal est évidemment sujette à critique et celle-ci doit être permanente. C'est là, d'après-moi, l'honneur comme la vocation des intellectuels que d'en proclamer la nécessité et de la pratiquer sans défaillance. Mais d'affirmer la suppression du conflit constant entre le bien et le mal, c'est professer une philosophie inhumaine, orgueilleuse et conduisant à des régimes tragiques pour la liberté de l'homme.

Sisyphe plutôt que Prométhée exprime mieux la philosophie qui inspire celui qui signe ces lignes. Mais il compte quand même le second parmi les grands symboles d'espérance de l'histoire humaine. 